\title{
Playing with Casimir in the vacuum sandbox
}

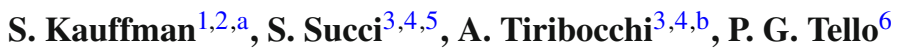 \\ ${ }^{1}$ The Institute for System Biology, Seattle, WA, USA \\ 2 Professor Emeritus, Biochemistry and Biophysics, The University of Pennsylvania, Philadelphia, PA, USA \\ ${ }^{3}$ Center for Life Nano Science@ La Sapienza, Istituto Italiano di Tecnologia, 00161 Rome, Italy \\ ${ }^{4}$ Istituto per le Applicazioni del Calcolo CNR, Via dei Taurini 19, Rome, Italy \\ ${ }^{5}$ Institute for Applied Computational Science, John A. Paulson School of Engineering and Applied Sciences, Harvard University, Cambridge, \\ USA \\ ${ }^{6}$ CERN, Geneva, Switzerland
}

Received: 20 April 2021 / Accepted: 9 October 2021 / Published online: 25 October 2021

(C) The Author(s) 2021

\begin{abstract}
The Casimir effect continues to be a subject of discussion regarding its relationship, or the lack of it, with the vacuum energy of fluctuating quantum fields. In this note, we propose a Gedankenexperiment considering an imaginary process similar to a vacuum fluctuation in a typical static Casimir set up. The thought experiment leads to intriguing conclusions regarding the minimum distance between the plates when approaching the Planck scale. More specifically, it is found that distance between the plates cannot reach a value below $\left(L / L_{P}\right)^{2 / 3}$ Planck lengths, being $L_{P}$ the Planck length and $L$ the typical lateral extension of the plates. Additional findings allow the conclusion that the approach between the two plates towards this minimum separation distance is asymptotic.
\end{abstract}

\section{Introduction and aim}

The physical vacuum seems to be a "busy place" described as "foamy" by Wheeler [1], and one of the most intriguing phenomena linked to it is the Casimir effect [2]. In this note, we propose a Gedankenexperiment based on the Casimir effect by following the tradition of speculating for the playful purpose of thinking through its consequences. This Gedankenexperiment is suggested with the caveat that the relation of the Casimir effect with the vacuum energy of fluctuating quantum fields is still open to debate at the time of this writing [3].

\footnotetext{
a e-mail: stukauffman@gmail.com (corresponding author)

b e-mail: adrianotiribocchi@gmail.com
}

\section{Gedankenexperiment setup}

The starting point is the typical configuration of the static Casimir effect: two uncharged, flat and perfectly conductive parallel plates in the vacuum, placed at a very small distance apart $d$ (typically in the order of microns or even a few nanometers).

Accordingly, the energy $E$ confined between the plates can be expressed as:

$E=-C \frac{\hbar c L^{2}}{d^{3}}$

Here $C=\pi / 24, c$ is the speed of light, $L$ is the lateral extension of the plates and the minus sign indicates a negative energy, corresponding to attraction between the plates. The Gedankenexperiment assumes the occurrence of a vacuum fluctuation between the plates (quantized by the value of $d$ ) subject to the energy-time relationship. The Casimir plates, initially separated at a distance $d$, will be therefore momentarily brought together to a smaller distance $d^{\prime}$, due to the fluctuation with energy $E_{n}=\hbar \omega_{n}$. Accordingly, considering such fluctuation, the variation $\Delta E$ of energy between the plates will be given by the difference between the initial value and the final one, with the former greater than the latter, since $C>0$ and $d^{\prime}<d$. Thus, it is possible to write:

$\Delta E=\hbar \omega_{n}=C \frac{\hbar c L^{2}}{d^{\prime 3}}-C \frac{\hbar c L^{2}}{d^{3}}$.

Let us consider now a standard interpretation of the energy-time indeterminacy relation which tells, in ordinary language, that if an amount of energy $\Delta E$ is "borrowed" from the vacuum, it must be "returned" quickly enough within a time interval $\Delta t$. In the context of this Gendankenexperiment, 
$\Delta t$ would correspond to the fluctuation lifetime. Mathematically, it is expressed by the energy-time uncertainty relation $\Delta E \Delta t \geq \hbar / 2$. Accordingly, $\Delta t$ is

$\Delta t \geq \frac{\hbar}{2 \Delta E}$

Taking into consideration Eq. (2), it is possible to write

$\Delta t \geq \frac{\hbar}{2 \Delta E}=\frac{1}{2 \omega_{n}}$

In this Gedankenexperiment, the boundary conditions assumed for the quantized mode between the plates allow $\omega_{n}$ to be expressed, for some integer number $n$, as:

$\omega_{n}=\frac{2 \pi n c}{d}$

Substituting Eq. (5) into Eq. (4) leads to the approximation

$\Delta t \sim \frac{d}{n c}$

At this point it is interesting to notice that, based on Eq. (2), the relationship between $d$ and $d^{\prime}$ takes the form

$\left(\frac{d^{\prime}}{d}\right)^{3}=\frac{1}{1+\frac{d^{2}}{d_{n}^{2}}}$,

with

$d_{n}=\sqrt{\frac{C}{2 \pi}} \frac{L}{n^{1 / 2}} \sim \frac{L / 7}{n^{1 / 2}}$.

Equation (7) can be also cast in a more compact for as follows:

$\frac{d^{\prime}}{d}=\frac{1}{\left(1+n \frac{d^{2}}{l^{2}}\right)^{1 / 3}}$,

where we have set $l \sim L / 7$. The expressions (7-9) constitute the cornerstone of the Gedankenexperiment, since they lead to a number of interesting considerations. The first one is that it shows the ratio $d^{\prime} / d$ as a function of the reduced separation $d / L$, depending parametrically on the wavenumber $n$. This dependence is plotted in Fig. 1 for different values of $n$. The graph indicates the existence of a characteristic wavenumber for each given value of the ratio $d / L$ below which the contraction of the space between the plates is really small. When approximating to such characteristic value of $n$, the ratio $d^{\prime} / d$ experiments a fast decrease, followed by a very slow decay with $d / L$ which is basically undetectable in the relevant regime $d / L \ll 1$. The second one is the emergence of two regimes which for convenience are identified here as

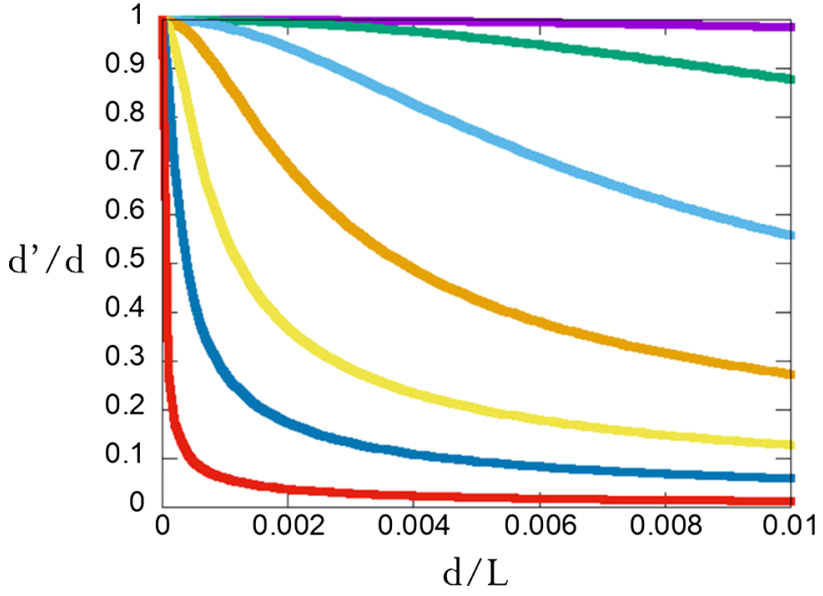

Fig. 1 The ratio $d^{\prime} / d$ as a function of the reduced separation $d / L$ for $n=10^{k}$ and with $k$ in the range from 1 to 8 (from top to bottom). The rapid drop of the ratio $d^{\prime} / d$ and subsequent saturation at increasing values of $n$ is clearly apparent. Note that $d / L$ is always well below 1 , as it should be within Casimir effect theory

"slow-mode" and "fast mode" which are subsequently analyzed in the following sections.

\section{Fast and slow-mode regimes}

Based on Eq. (9), two regimes can be identified: (i) "slowmode", characterized by

$n \frac{d^{2}}{l^{2}} \ll 1$

and (ii) "fast mode", characterized by the opposite inequality. The slow-mode inequality given by Eq. (10) implies that at each given value of the reduced separation $d / L$ there is a maximum wavenumber, which we call $n_{1}$, such that the ratio $d / d^{\prime}$ is slightly below one, therefore corresponding to small contractions between the plates. By definition then:

$n_{1}=\frac{l^{2}}{d^{2}}$

In the slow-mode regime $n \ll n_{1}$, Eq. (9) reduces to:

$\frac{d^{\prime}}{d}=1-\frac{n}{3} \frac{d^{2}}{l^{2}}=1-\frac{1}{3} \frac{n}{n_{1}}$,

which, by definition, is just slightly smaller than one.

In the fast-mode regime, Eq. (9) leads to:

$\frac{d^{\prime}}{d} \sim n^{-1 / 3}\left(\frac{l}{d}\right)^{2 / 3}=\left(\frac{n}{n_{1}}\right)^{1 / 3}$ 
This expression shows that $d^{\prime}$ decreases very slowly with the wavenumber $n$ and cannot reach the value zero for any finite value of $n$. In other words, the separation between the plates cannot be zero. We shall return to this point in the final part of this paper, when discussing the implication of the Eq. (13) in the limit of the Planck scale.

At this point, it is worth noting that as required by the Casimir effect theoretical formalism, the ratio $d / L$ must be necessarily much smaller than one for Eq. (1) to hold. Hence, as an example, we consider a ratio $d / L=10^{-3}$ which thus gives $n_{1} \sim 10^{4}$. Based on the Eqs. (9) and (11), Eq. (6) leads to

$$
\Delta t=\frac{d^{\prime}}{n c}\left(1+10^{-4} n\right)^{1 / 3} .
$$

As it indicates, the quantity in brackets only becomes significant for wave numbers $n>10^{4}$. It should be noted that, considering the "slow mode regime" with $d=1 \mathrm{~nm}$ and $c \sim 10^{8} \mathrm{~m} / \mathrm{s}$, it gives $d / c$ of the order of the attosecond $\left(10^{-18} s\right)$. This means that only modes with $n \ll 10^{4}$ could be possibly detected within the current frontier of ultrafast technology. This is analyzed further in the following sections.

\subsection{Analysis of the slow-mode regime}

Considering $L / d \sim 10^{3}, n_{1} \sim 10^{4}$, hence for $n<n_{1}$ ("slow modes"), expanding the Eq. (12) leads to:

$$
\frac{d^{\prime}-d}{d} \simeq-\frac{n}{3} 10^{-4},
$$

which indicates a relative contraction in the order of the $4 \mathrm{th}$ digit for $n \sim 10$, a measurable effect as long as such measurement is performed within the limits of the fluctuation lifetime $\Delta t$. Since, in this regime, $d^{\prime} \simeq d$, one has

$$
\Delta t \sim \frac{d}{c n} .
$$

Given that the maximum value of $n$ in the slow mode regime is $n_{1}=10^{4}$, and approximating $c \sim 10^{8} \mathrm{~m} / \mathrm{s}$, we obtain a measuring time of $10^{-21} \mathrm{~s}$, which is between two and three orders of magnitude beyond the current technological possibilities even for measurement of ultrafast processes [4].

\subsection{Analysis of the fast-mode regime}

In the fast-mode regime, Eq. (13) leads to

$$
\frac{d^{\prime}}{d} \simeq\left(\frac{10^{4}}{n}\right)^{1 / 3} \simeq 10 n^{-1 / 3} .
$$

This shows that, due to the small exponent $-1 / 3$, it takes very large wavenumbers $n$ to achieve substantial contrac- tions. For instance, for $n=10^{6}$ a contraction is obtained of $d^{\prime}=d / 10$, and for $n=10^{9}$, it gives $d^{\prime}=d / 100$. Note that the limit $n \rightarrow \infty$ is unphysical since the wavelength of the photon cannot be lower than the Planck length $L_{p}$. This is discussed more in detail in the next paragraph. Moreover, as it is readily seen from Eq. (14), the corresponding fluctuation lifetime $\Delta t$ is of the order of $10^{-24}$ and $10^{-27}$ s respectively, hence far outside reach of the present technology.

In summary, we have seen that a "vacuum fluctuation" between the plates, both of a high energetic mode $\left(n>10^{4}\right)$ or a low energetic mode $\left(n<10^{4}\right)$, produces a separation effect between the plates that is beyond experimental reach with today's technological capability. Perhaps a challenge is left for future experimental instrumentation developments. The following observation is relevant at this point. The single photon assumed to be extracted from within the plates, being a single mode, has an energy which does not grow with the transverse area $L^{2}$ of the plates, while the energy in between the plates does (see Eq. (1)). Accordingly, in order to compensate for this different scaling and in principle obtaining a measurable effect for $\Delta E$ and, therefore, on the distance variation between them, a highly energetic mode is needed, which then becomes unmeasurable within the associated extremely short lifetime $\Delta t$ of its corresponding fluctuation. On the other hand, assuming a photon with reasonable energies will eventually result in an undetectable displacement of the walls.

\section{Down to the Planck scale}

In this section, the authors would like to take this Gedankenexperiment further and playfully speculate about the results obtained. In order to explore this matter, let us introduce the Planck wavenumber $n_{P}$, as the value of $n$ such $\Delta t=t_{P}$ and $\lambda=L_{P}$, meaning the Planck time and the Planck wavelength respectively, $L_{P}$ being the Planck length. By definition, we have:

$n_{P}=\frac{2 d}{L_{P}}$.

The Eq. (13) becomes minimum assuming the largest possible value for $n$, given by $n_{P}$. Using the definitions given by Eqs. (11) and (18), we obtain:

$d_{\text {min }}=d\left(\frac{n_{1}}{n_{P}}\right)^{1 / 3} \sim L^{2 / 3} L_{P}^{1 / 3}$.

This shows that even upon considering the most energetic possible mode (a Planckian photon), the "Casimir space" cannot contract below $\left(L / L_{P}\right)^{2 / 3}$ Planck lengths. This result is intriguing, for it singles out a minimum length scale well 
above the Planck length. Thus, the authors dare speculating that, although with an exchange of exponents between $L$ and $L_{P}$, an intriguing analogy holds with the holographic expression $L_{\min }=L_{P}^{2 / 3} L^{1 / 3}$. As is well known, this expression derives from assuming that the relevant quantum gravitational degrees of freedom associated with a given spatial region are located on the surface and not on the volume $[5,6]$. The relation 19 might also suggest a dependence of the boundary conditions of our Gedankenexperiment.

Coming back to the thought experiment and starting with Eq. (14),

$\Delta t \sim \frac{1}{n c} d^{\prime}\left(1+10^{-4} n\right)^{1 / 3}$,

when considering $n=n_{P}$, this approximates to

$\Delta t \sim \frac{d^{\prime}}{c} \frac{1}{10 n_{P}^{2 / 3}}$.

By taking into account the definition of $n_{P}$, it leads to:

$\Delta t_{P} \sim \frac{d^{\prime}}{10 c}\left(\frac{L_{P}}{d}\right)^{2 / 3}$.

By considering Eq. (13) and after some algebra, it is possible to arrive, as expected, to:

$\Delta t_{P} \sim \frac{n_{1}^{1 / 3}}{c} L_{P} \sim \frac{L_{P}}{c} \sim t_{P}$.

Note that $\Delta t_{P}$ as computed above is much smaller than the smallest time interval supported by the hypothetical time allowed by the Casimir theoretical formulation

$t_{\text {min }}=\frac{d_{\text {min }}}{c}=\left(\frac{L}{c}\right)^{2 / 3} t_{P}^{1 / 3}$.

This might suggest some connection with a generalized Heisenberg principle and more particularly given the case that, in our Gendankenexperiment, the distance between the plates cannot be made arbitrarily small. More concretely smaller than the Planck length [7]. As a matter of fact, it is interesting, as a final consideration of this note, relating the energy between the plates with respect to the Planck energy, $E_{P}$. Starting with Eq. (1) and taking into account Eq. (19), we obtain:

$E_{\text {min }} \simeq-\frac{\hbar c L^{2}}{d_{\text {min }}^{3}}=-\frac{\hbar c}{L_{P}}=-E_{P}$,

where we have assumed $|E(d)| \ll E_{P}$ as it is appropriate for $d>d_{\min }$. This result would seem paradoxical because, considering a fluctuation of the order of the Planck energy, would imply that $E=-2 E_{p}$, which cannot be since $E$ is a free parameter. This apparent paradox is explained by considering that in fact $d^{\prime}$ approximates only asymptotically to $d_{\text {min }}$, never to reach exactly this limiting value as expressed in Eq. (19). More concretely, by taking Eq. (2) with $C=1$, we compute:

$E\left(d=L_{P}\right)=\frac{\hbar c L^{2}}{L_{P}^{3}}=E_{P}\left(\frac{L}{L_{P}}\right)^{2} \gg E_{P}$.

As this expression suggests, the apparent paradox mentioned above results from the fact the energy between the plates is singular as $d$ approaches zero, hence it takes much more energy than $E_{P}$ to push $d^{\prime}$ from $d_{\min }$ down to $L_{P}$. The interesting conclusion is that while $E\left(d^{\prime}\right)$ closely approaches $E\left(d_{\min }\right), d_{\text {min }}$ still remains far above the Planck length.

\section{Conclusions}

Our excursion into the Casimir sandbox through the thought experiment proposed in this note leads to the following observations:

1. Considering the occurrence of a hypothetical "vacuum fluctuation" between the plates subject to the energy-time uncertainty leads to two differentiated regimes ruled by the scaling relationship $L / d=10^{3}$ between the lateral dimensions of the plates $L$ and their distance $d$, characterized by the value of the mode wave number $n$. One which we will call "fast mode" where $n>10^{4}$ and another one which we will call "slow mode" where $n<10^{4}$.

2. The potential shrinking effect between the plates of both, the high energetic mode $\left(n>10^{4}\right)$ or the low energetic mode $\left(n<10^{4}\right)$, cannot be measured with today's technological capability. Perhaps, in the low energetic mode, future advances in technology might be able to cope with the challenge.

3. By considering a fluctuation at the order of the Planck energy, the momentarily contracted distance between the plates cannot reach a value $d_{\text {min }}$ below $\left(L / L_{P}\right)^{2 / 3}$ Planck lengths. More precisely, $d_{\min }=L^{2 / 3} L_{P}^{1 / 3}, L$ being the lateral extension of the plates and $L_{P}$ the Planck distance. The authors noticed the intriguing analogy between the expression for $d_{\min }$ and the one $L_{\min }=L_{P}^{2 / 3} L^{1 / 3}$ which derives from assuming that the relevant degrees of freedom of quantum gravity are located on the surface and not on the volume ("holographic principle").

4. If the diminished distance $d^{\prime}$ between the plates, following the thought experiment, approaches the minimum distance $d_{\min }$, it does it asymptotically. This leads to the interesting observation that while the energy between the plates, $E\left(d^{\prime}\right)$, approaches the minimum one, $E\left(d_{\min }\right)=$ 
$E_{P}$, the minimum Casimir distance $d_{\min }$ remains far above the Planck length, $L_{P}$. Conversely, the Casimir energy at the Planck scale exceeds the Planck energy by a factor $\left(L / L_{P}\right)^{2 / 3}$.

5. One may finally wonder whether the present analysis would hold if the geometry of the conducting plates is not flat. Although this is beyond the scope of this manuscript, it is interesting to note that modifying the shape of the plates can turn the sign of the energy from negative to positive, thus leading to a repulsive rather than an attractive interaction [8]. This has been shown, for example, in Ref. [9], where a small elongated metal particle in vacuum is subject to a repulsive force when centered above a metal plate with a hole. Repulsive long-range forces, of quantum electrodynamic origin, have been also measured between materials with suitable optical properties and immersed in a fluid [10]. Even more intriguing is the realm of soft condensed matter, where Casimir-like forces have been found to act between surfaces immersed in a binary fluid close to its critical point. Such forces, caused by the fluctuations of the concentration (whose relevant scale is $k_{B} T$, where $k_{B}$ is the Boltzmann constant and $T$ the temperature) within the fluid film separating the surfaces, can be either attractive or repulsive, depending on the adsorption preference of the fluid in contact with the solid body [11,12]. Finally, it is known that near-contact forces between macroscopic bodies play a major role on the rheology of soft materials [13].

Acknowledgements All authors warmly acknowledge Andrea Gambassi for useful discussions and a critical reading of the manuscript. SS and AT would like to acknowledge funding from the European Research Council under the Horizon 2020 Programme Grant Agreement n. 739964 ("COPMAT"). This document has the CERN Open reference CERN-OPEN-2021-002.

Data Availability Statement This manuscript has no associated data or the data will not be deposited. [Authors' comment: All data generated in this study are included in this published article.]

Open Access This article is licensed under a Creative Commons Attribution 4.0 International License, which permits use, sharing, adaptation, distribution and reproduction in any medium or format, as long as you give appropriate credit to the original author(s) and the source, provide a link to the Creative Commons licence, and indicate if changes were made. The images or other third party material in this article are included in the article's Creative Commons licence, unless indicated otherwise in a credit line to the material. If material is not included in the article's Creative Commons licence and your intended use is not permitted by statutory regulation or exceeds the permitted use, you will need to obtain permission directly from the copyright holder. To view a copy of this licence, visit http://creativecomm ons.org/licenses/by/4.0/.

Funded by SCOAP $^{3}$.

\section{References}

1. J.A. Wheeler, On the nature of quantum geometrodynamics. Ann. Phys. 2(6), 604-614 (1957)

2. H.B.G. Casimir, On the attraction between two perfectly conducting plates. Proc. Kon. Ned. Akad. Wet. 51, 793 (1948)

3. R.L. Jaffe, Casimir effect and the quantum vacuum. Phys. Rev. D 72, 021301(R) (2005)

4. S. Grundmann, Zeptosecond birth time delay in molecular photoionization. Science 370(6514), 339-341 (2020)

5. C.R. Stephens, G. 't Hooft, B.F. Whiting, Black hole evaporation without information loss. Class. Quantum Gravity 11(3), 621-648 (1994)

6. L. Susskind, The world as a hologram. J. Math. Phys. 36(11), 63776396 (1995)

7. A. Kempf et al., Hilbert space representation of the minimal length uncertainty relation. Phys. Rev. D 52, 1108-1118 (1995)

8. Q.-D. Jiang, F. Wilczek, Chiral Casimir forces: repulsive, enhanced, tunable. Phys. Rev. B 99, 125403 (2019)

9. M. Levin, A.P. McCauley, A.W. Rodriguez, M.T.H. Reid, S.G. Johnson, Phys. Rev. Lett. 105, 090403 (2010)

10. J.N. Munday, F. Capasso, V.A. Parsegian, Measured long-range repulsive Casimir-Lifshitz forces. Nature 457, 170-173 (2009)

11. C. Hertlein, L. Helden, A. Gambassi, S. Dietrich, C. Bechinger, Direct measurement of critical Casimir forces. Nature 451, 172175 (2008)

12. A. Gambassi, The Casimir effect: from quantum to critical fluctuations. J. Phys. Conf. Ser. 161, 012037 (2009)

13. A. Montessori, M. Lauricella, N. Tirelli, S. Succi, Mesoscale modelling of near-contact interactions for complex flowing interfaces. J. Fluid. Mech. 872, 327-347 (2019) 